\title{
Rezensionen / Reviews
}

Tobies, Renate, Hg., 2008. „Aller Männerkultur zum Trotz". Frauen in Mathematik, Naturwissenschaften und Technik. Frankfurt a. M./New York: Campus (2., überarb. und erw. Auflage), brosch. 364 S., $32,90 €$, ISBN-13: 978-3593386140.

Vogt, Annette, 2007. Vom Hintereingang zum Hauptportal? Lise Meitner und ihre Kolleginnen an der Berliner Universität und in der Kaiser-Wilhelm-Gesellschaft. Stuttgart: Steiner Verlag (=Pallas Athene. Beiträge zur Universitäts- und Wissenschaftsgeschichte, 17), geb. 550 S., $84 €$, ISBN-13: 978-3515088817.

$\mathrm{Zu}$ den ertragreichsten Forschungssträngen der wissenschaftshistorischen Frauen- und Geschlechterforschung in den letzten Jahren zählen solche Ansätze, die Lebens- und Institutionsgeschichten quellennah erforschen und diese Ergebnisse unter analytischen Perspektiven zusammenfügen. Die hier zu besprechenden Bücher, wiewohl ganz unterschiedlichen Charakters, fallen beide in diese Kategorie. Beide breiten vor der Leserin beziehungsweise dem Leser eine Fülle von Namen, Institutionen und Konstellationen aus und fügen sie in größere Muster ein.

Der von Renate Tobies herausgegebene Aufsatzband erscheint in zweiter Auflage und ist gegenüber der Fassung von 1997 erheblich verändert. Im Untertitel ist nun auch von Frauen in der Technik die Rede. Dieser Anspruch wird insbesondere mit einem neu hinzugekommenen Beitrag der Herausgeberin über „Forscher/innen in der elektrotechnischen Industrie vor 1945" eingelöst, in dem sie sich mit der für die Industrieforschung typischen Transdisziplinarität von Forschungsszenarien beschäftigt. Hinzugekommen ist auch ein Exkurs über Mathematikerinnen in der Luftfahrtforschung. Wenn dieser mit der These eingeleitet wird, „dass es kein mögliches Berufsfeld gibt, das Wissenschaftlerinnen nicht besetzten“ (S. 115), dann klingt hier der emanzipatorische Impetus an, der dieses Buch von Grund auf prägt: aufzuzeigen, dass Frauen „aller Männerkultur zum Trotz“ erfolgreich in Mathematik, Naturwissenschaften und Technik tätig waren und tätig sein können. Dies ist nicht nur ein bis heute aktuelles Thema, sondern auch im Blick 
auf die ungebrochene Flut mehr oder weniger wissenschaftlich auftretender Publikationen über Geschlechterdifferenzen, in denen immer wieder auch historisch - von jeglicher Sachkenntnis unbeschwert - argumentiert wird. Hier ist in der Tat Aufklärungsarbeit vonnöten.

Wer aufklären will, muss zunächst selbst verstehen. Und so gilt dem Verständnis der entscheidenden Faktoren, dem Aufzeigen der begünstigenden und hemmenden Einflüsse für die weibliche Partizipation an Mathematik und Naturwissenschaften das besondere Interesse der Autorinnen und Autoren des Sammelbands. Im einführenden und mit Abstand längsten Beitrag formuliert die Herausgeberin dazu zehn Thesen, die in einem weit ausgreifenden Forschungsüberblick entfaltet werden. Ebenso wie die zugehörige fünfzehn Seiten umfassende Bibliographie wurde dieser gegenüber der Erstauflage auf den neuesten Stand gebracht. Übernommen wurden dagegen die Beiträge von Lorraine Daston über die Quantifizierung der weiblichen Intelligenz, von der Herausgeberin über „Mathematiker/innen und ihre Doktorväter“, von Waltraud Voss über die Mathematikerinnen Johanna und Gertrud Wiegandt und von Irene Pieper-Seier über die Mathematikerin Ruth Moufang. Die Dominanz der Mathematikgeschichte wird in der neuen Auflage noch gesteigert durch einen gemeinsamen Beitrag von Mechthild Koreuber und der Herausgeberin über Emmy Noether, die als erste Frau in der Mathematik eine eigene wissenschaftliche Schule ausbildete, sowie durch den Aufsatz von Christa Binder über die Zahlentheoretikerin Olga Taussky-Todd. Die bereits in der ersten Auflage enthaltenen Beiträge von Annette Vogt über Frauen als Abteilungsleiterinnen an Kaiser-WilhelmInstituten, von Ute Deichmann über Frauen in der Genetik und von Jeffrey Johnson über Frauen in der chemischen Industrie komplettieren den Band. Fast alle Beiträge muten also biographisch orientiert an, und in der Tat lernt man durch die Lektüre des Bandes viele Wissenschaftlerinnen kennen. Immer wieder aber werden diese Lebensgeschichten auf die größeren strukturellen Fragen - etwa die disziplinäre Stellung des Forschungsgebietes, die Rolle des Doktorvaters oder die zeitgenössischen politischen Entwicklungen - rückbezogen. Offensichtlich erfolgte hier eine intensive Abstimmung, die dem Band eine ausgeprägte Kohärenz verleiht und seine Lektüre auch für all diejenigen lohnend macht, die sich für das große Bild jenseits der Einzelschicksale interessieren. Der Personenindex erleichtert die Vernetzung der verschiedenen Beiträge.

Problematisch erscheint hingegen die in der Neuauflage noch einmal verschärfte Dominanz der mathematikhistorischen Beiträge, zumal diese sich in einigen Teilen den Leserinnen und Lesern ohne mathematische Vorkenntnisse kaum erschließen werden. Hier wäre die Verengung (auf Mathematik) statt Erweiterung (auf Technik) des thematischen Rahmens eine Alternative gewesen, zumal es sich bei den hinzugekommenen Frauen in der Industrie nicht selten gerade um ausgebildete Mathematikerinnen 
gehandelt hat. Für Institutsbibliotheken, die den Band in der ersten Auflage schon besitzen, lohnt sich wegen der inhaltlichen Verschiebungen und Fortschreibungen durchaus die Anschaffung der zweiten Auflage; für die private Kaufentscheidung dürfte in jedem Fall das Interesse an den alten und neuen mathematikhistorischen Beiträgen ausschlaggebend sein.

Ist Aufklärung das Ziel von Tobies' Sammelband, so ist Erinnerung das Anliegen von Annette Vogts monumentaler Längsschnittstudie über die Wissenschaftlerinnen an der Berliner Universität und in der Kaiser-Wilhelm-Gesellschaft bis circa 1955. Erinnerung an all jene Frauen, die in diesen Institutionen forschten und lehrten und in vielen Fällen kaum Spuren hinterlassen haben. Erinnerung aber auch an diejenigen, die von den Nationalsozialisten vertrieben, verfolgt und gar getötet wurden. Dieses Anliegen zeigt sich vor allem in den Kapiteln über Verfolgung und Widerstand, in denen sehr kritische Blicke auf die unmittelbare deutsche Nachkriegsgeschichte eröffnet werden. Die detaillierten Angaben über widerständige Wissenschaftler und ihre solidarischen Handlungen gegenüber Verfolgten werfen aber auch ein anderes Licht etwa auf Otto Hahn, der als Kollege der gerade noch ins Exil sich rettenden Lise Meitner durch die Meitnerliteratur mitunter in recht düsteren Farben porträtiert worden ist. Annette Vogt spricht hier mit der ganzen Autorität derjenigen, die sich über die Jahre in die Quellen und Spuren hineingelesen hat und öffnet uns neue Blicke auf alte Bekannte.

Dieses Buch ist, mit einem nicht übersetzbaren englischen Ausdruck gesagt, „a labour of love“, eine Arbeit, wie sie nur mit großem und ausdauerndem Engagement und Herzblut zustande kommen kann. Das imposante, über fünfzig Seiten umfassende Literaturverzeichnis ist, wie eine Stichprobe ergibt, noch nicht einmal vollständig und gibt Zeugnis von der Fülle an Informationen und Hinweisen, die die Autorin über die Jahre gesammelt hat. Vielleicht das wichtigste Werkzeug zu ihrer Erschließung ist der beigefügte Personenindex, der es erlaubt, Informationen zu ein und derselben Person aus verschiedenen Kapiteln zusammenzufügen. Denn in Vogts Studie tauchen die Wissenschaftlerinnen nicht namentlich in den Überschriften der Kapitel und Abschnitte auf. Gegliedert ist das Buch vielmehr chronologisch, indem es den politischen Zäsuren und Systemen - Kaiserreich, Weimarer Republik, Nationalsozialismus, Nachkriegszeit folgt, in die es jeweils auch in frauengeschichtlicher Perspektive kompakt und kompetent einführt. Dieses Verfahren sorgt im Verlauf der Studie für eine gewisse Redundanz in der Nennung von Personen und Institutionen, es hat aber den großen Vorteil, dass sich die einzelnen Abschnitte unabhängig voneinander lesen und verstehen lassen, man sich also gezielt über einzelne Perioden und Entwicklungen kundig machen kann. Möchte man hingegen das Schicksal einer bestimmten Person durch die Zeitabschnitte verfolgen, so öffnet der Index problemlos den Zugang zu allen zugehörigen 
Textpassagen. Der Vorrang der institutionell-strukturgeschichtlichen vor der biographischen Gliederung erscheint damit als der bestmögliche $\mathrm{Zu}$ gang zu der Fülle von verarbeiteten biographischen und institutionengeschichtlichen Daten.

Zentrales Analyseinstrument ist die von ihrem Geburtsjahrgang abhängige Gruppierung der Wissenschaftlerinnen in drei Alterskohorten oder "Generationen“. Die - in der Wissenschaftsgeschichte noch viel zu wenig gestellte - Frage nach dem Einfluss des Alters auf die Folgen der nationalsozialistischen Vertreibung (oder anderer Ereignisse) für die Karriereverläufe zeigt, dass sich die Biographien innerhalb einer Generation ähneln und von denen der älteren oder jüngeren Wissenschaftlerinnen deutlich abheben. Dabei dient der Autorin die Unterscheidung zwischen - abgelehnter - Außenseiterin und - positiv besetzter - Ausnahme als semantische Leitperspektive, anhand derer sie die Oszillation von der Ablehnung zu einer gewissen Normalität des Wissenschaftlerinnen-Daseins und zurück zur Verdrängung (bis in die Nachkriegszeit hinein) ebenso nachzeichnet wie die Chancen, die sich den Frauen durch die Kriege eröffneten.

Vogts Studie ist als Vergleich zweier wissenschaftlicher Institutionen in der deutschen Reichshauptstadt angelegt, der Berliner Universität und der Kaiser-Wilhelm-Gesellschaft. Dieses Konzept ermöglicht es, alle in Berlin tätigen Wissenschaftlerinnen zu erfassen und die ,Frauenfreundlichkeit' von Universität und außeruniversitärer Forschung vergleichend zu bewerten. Zugleich werden mit dieser Ausrichtung der Untersuchung aber auch drängende Fragen außer Acht gelassen, nämlich diejenigen nach Beziehungen und möglichen Synergieeffekten zwischen beiden Arbeitsfeldern. Dass in Berlin mehr Frauen als an jeder anderen Universität promovierten und habilitierten, mag neben der absoluten Größe gerade durch die KaiserWilhelm-Institute mit ihren Arbeitsmöglichkeiten bedingt sein. Die zahlreichen Wissenschaftlerinnen, denen wir im Verlauf des Buches an beiden Institutionen begegnen - die im Untertitel explizit genannte Lise Meitner steht hier geradezu paradigmatisch - sind dafür ein deutlicher Hinweis. Wichtig wäre also nun gerade der Vergleich mit einer anderen Universität, etwa Bonn, Göttingen oder Heidelberg, um die Vermutung der kausalen Abhängigkeit des hohen Anteils an Wissenschaftlerinnen von der großen Zahl wissenschaftlicher Institutionen und KWI-Institute überprüfen zu können. Dass die Autorin diese Aufgabe nicht auch noch geschultert hat, mag man ihr freilich angesichts der Fülle des verarbeiteten Materials ebenso wenig vorwerfen wie den Umstand, dass an vielen Stellen weitergehende Vergleiche mit männlichen Kontrollgruppen wünschenswert wären. Mit ihrem Buch hat Annette Vogt eine Grundlage geschaffen, auf der solche weiterführenden Studien aufbauen können und das Erinnern neben dem Aufklären gedeihen kann.

Beate Ceranski, Stuttgart 


\section{Hoffmann, Christoph, 2006. Unter Beobachtung. Naturforschung in der Zeit der Sinnesapparate. Göttingen: Wallstein Verlag (=Wis- senschaftsgeschichte), 252 S., $39 €$ €, ISBN-13: 978-3-8353-0075-0.}

Während die Praxis des Experimentierens mittlerweile einen fest etablierten Platz in der Historiographie der Wissenschaften hat und als treibende Kraft der Wissensproduktion anerkannt ist, wird die Praxis des Beobachtens immer noch wenig gewürdigt. Für Ian Hacking etwa ist sie von nur geringer Bedeutung. An dieser Stelle setzt Christoph Hoffmann mit seiner Studie ein. Denn wenn auch in der Epoche selbstaufzeichnender Apparate die Beobachtung nicht mehr ist als ein Akt der Information, so war das doch nicht immer so: Zumindest in der Epoche der Naturforschung bedeutete Beobachten „in das Resultat der Beobachtung konstitutiv verwickelt zu sein“ (S. 29).

Hoffmanns Untersuchung beginnt mit jener Zeit, in der eine auf „civility“ gegründete Praxis der Anerkennung von Beobachtungsdaten, wie sie von Steven Shapin untersucht wurde, durch ein neues „Regime der Beobachtung" abgelöst wird. Zu Beginn des 18. Jahrhunderts war nicht mehr der soziale Status des Beobachters der Garant für die Richtigkeit seiner Daten, sondern die möglichst genaue Befolgung von Vorschriften. Beobachtung, so Hoffmann, wurde nicht als Quelle von Erfahrung, sondern „als Gegenstand der Regulierung“ (S. 37) zum Objekt wissenschaftlicher Aufmerksamkeit. Erxleben oder Senebier etwa analysierten mit ihren Anweisungen keineswegs die Bedingungen eines Beobachtungsvorganges. Vielmehr legten sie fest, wie die Handhabung von Apparaten und Sinnen erfolgen sollte.

Wie die irreduzible Materialität der Bedingungen schließlich hervorbrechen sollte zeigt der Autor am Beispiel von Jean-André de Lucs Recherches sur les modifications de l'atmosphère. In diesem 1772 erschienenen Buch materialisierte sich gewissermaßen das Wuchern einer Forschung, die sich rigoros sämtlichen Bedingungen der Beobachtungstätigkeit widmen wollte. De Lucs Detailversessenheit führte nicht nur dazu, dass die formale Einheit des Werkes durch einen Wust an Beobachtungsdaten gesprengt wurde, sondern auch zum Verlust des Paradigmas der Vervollkommnung: Instrumente waren nicht länger durch Kontrolle und Verfahrensregeln zu verbessern. Stattdessen galt es, die ihnen eigenen Unvollkommenheiten in Betracht zu ziehen. Wie Hoffmann feststellt, überwand die Untersuchung des Instruments nicht die Grenzen seiner Materialität, sondern sie brachte diese Materialität als Gegenstand des Wahrnehmens, Denkens und Handelns überhaupt erst hervor.

Daran anschließend entwickelt Hoffmann detailgenau die Geschichte einer berühmten Feststellung, nämlich jener Bemerkung des Astronomen Friedrich Wilhelm Bessel, „daß zwischen zwey Beobachtern ein unwillkürlicher beständiger Unterschied Statt finden kann" (S. 134). Während in der 
Astronomie- und Psychologiegeschichte geglaubt wurde, hier sei die Entdeckung der „persönlichen Gleichung“ zu erkennen, also jene spezifische Reaktionszeit, durch die sich ein Beobachter vom anderen unterscheidet, so zeigt Hoffmann, dass sich die Situation wesentlich komplizierter darstellte. Denn bis zur ersten Hälfte des 19. Jahrhunderts war die Differenz der Beobachter kein Gegenstand weiterer Forschungen. Tradiert wurde sie nicht als wissenschaftliches Objekt, sondern als operative Forderung, das heißt als etwas, das nur im Umgang mit den Apparaten und im konkreten Vollzug von Verfahren Aufmerksamkeit fand.

Hoffmanns erklärtes Ziel ist es, sich der Verführungskraft pauschaler Thesen über Subjektivität und Objektivität und über das Verhältnis von Apparaten und Subjekten entgegen zu stellen. Statt ihrer fordert - und verwirklicht - er eine Geschichtsschreibung, die sich den „widerspenstigen Details“ (S. 276) widmet und damit den kleinen Verschiebungen, Verwicklungen und Widersprüchen genauso viel Raum bietet wie den großen Umbrüchen und Neuordnungen. Manchmal hätte man sich gewünscht, etwas mehr über den Kontext der beschriebenen Beobachtungsregimes zu erfahren, um etwa zu verstehen, was das für eine Astronomie war, in der Genauigkeit eine so wichtige Rolle spielte. Dass es um 1800 auch andere Verfahrensweisen gab, zeigt sich beispielsweise an der Verwunderung Maskelyns über die Ungenauigkeit der Herschel'schen Beobachtungen, die eben weniger auf Positionsbestimmungen als vielmehr auf eine naturgeschichtliche Beschreibung der Himmelskörper zielte.

Die Lektüre von Christoph Hoffmanns Buch ist jedem zu empfehlen, der sich für die epistemischen Probleme der Beobachtung interessiert: Es ist nicht nur selbst ein gutes Beispiel für genaue Beobachtung, es ist auch ein Fundus an präzisen Thesen und Reflexionen zu verschiedenen Themen der heutigen Epistemologie.

Thomas Brandstetter, Wien

Rentetzi, Maria, 2009. Trafficking Materials and Gendered Experimental Practices. Radium Research in Early 20th Century Vienna. New York: Columbia University Press, geb. 320 S., 51,99 €, ISBN-13: 978-0-231-13558-0.

Das Jahr 1910 bescherte der Stadt Wien das Institut für Radiumforschung, das neben Paris, Cambridge und (später) Berlin schnell zu einem der bedeutendsten Zentren der Radioaktivitätsforschung aufstieg. Eine wichtige Rolle spielte dabei das Objekt der Forschung selbst: Radium. Als nicht leicht zugängliche Ware hatte es seinen Preis. Entscheidend für die Wiener war deshalb der privilegierte Zugang, den sie - jedenfalls in den letzten Jahren der österreichischen Monarchie - zu den böhmischen Erzgruben hatten. Nicht 
zuletzt dies begründete die Bedeutung des Radiuminstituts in diesem jungen, interdisziplinären Forschungsfeld. Eine weitere Voraussetzung war, dass sich das Institut zu einem attraktiven Arbeitsumfeld für studierte Frauen entwickelte.

Maria Rentetzis Geschichte der Radioaktivität handelt von solchen bislang wenig thematisierten Bedingungen wissenschaftlichen Arbeitens. Am Beispiel des Wiener Instituts für Radiumforschung untersucht sie die sozialen und materiellen Bedingungen der Radioaktivitätsforschung von ihren Anfängen bis in die Mitte des 20. Jahrhunderts. Dies ist aufschlussreich, weil Rentetzi die Radioaktivitätsforschung und die "radioactive people“ aus der Eindimensionalität einer allzu stark an der Entwicklung von Disziplinen orientierten Wissenschaftsgeschichte befreit. Radioaktivitätsforschung, so zeigt Rentetzi, war von Anfang an eingespannt in politische Projekte, sie war abhängig von einem Markt für Radium und von der konkreten Ausprägung des akademischen und intellektuellen Lebens, sie wurde beeinflusst von Architektur und von sozialen Rollenbildern. Und umgekehrt prägte der radioaktive Stoff seine soziale Umwelt und nicht zuletzt den Umstand, dass Frauen im Wiener Institut über die sonst derzeit üblichen Tätigkeiten als Erbsenzählerinnen und ähnliches hinaus eine aktive Rolle in der Forschung spielen konnten.

„Trafficking materials“ ist eine Stoffgeschichte, die das Material der Forschung unter Rekurs auf anthropological and cultural studies betrachtet, ein Konzept, das auf der Linie von Klein und Lefèvres Materials in EighteenthCentury Science. A Historical Ontology (2007) die Geschichte wissenschaftlicher Materialien fortschreibt. Als „trafficking material“ besaß Radium die Eigenschaft, dass seine Zirkulation als Ware sowie als wissenschaftliches und medizinisches Objekt zu einem untrennbaren Teil seiner Identität wurde. Dabei ist die entscheidende Erkenntnis, die Rentetzi an verschiedenen Beispielen belegt, dass diese Bewegung zugleich soziale Effekte zeitigte. Das Konzept lässt sich auf andere Ressourcen wissenschaftlichen Arbeitens übertragen, etwa auf Versuchstiere in Biologie und Medizin. Aber anders als Latours immutable mobiles fordert es dazu heraus, eine weniger wissenschaftszentrierte Geschichte eines wissenschaftlichen Objekts zu schreiben. Eine umfassende „kulturelle Biographie“ des Radiums kann Rentetzi selbst aber nur anreißen, da sich ihre Erzählung auf die facettenreiche Geschichte des Instituts für Radiumforschung konzentriert.

In drei ausführlichen Kapiteln entfaltet die Autorin ausgehend von der institutionellen und personellen Geschichte des Instituts für Radiumforschung eine architektonische, soziale und politische Geographie Wiens. Im Zentrum stehen dabei die Frauen des Instituts und die These, dass sich ihr hoher Anteil am Institutspersonal aus einem Bündel sozialer, kultureller und teils materieller Bedingungen erklärt. Die Untersuchung wandelt sich damit streckenweise zu einer kenntnisreichen Lokalstudie des Wiener akade- 
mischen Kontexts, des liberalen und sozialdemokratischen Denkens und der Emanzipation der Frauen im akademischen Leben Wiens. Eine zentrale Rolle spielte das sogenannte Medizinerviertel als Kern einer produktiven wissenschaftlichen Kultur. Einen klaren Bruch mit dieser ideal erscheinenden Wissenschaftskultur - deren problematische Seiten kürzlich etwa Baader/ Hofer/Mayer in Eugenik in Österreich (2007) thematisiert haben - markieren der Austrofaschismus und der Anschluss an das nationalsozialistische Deutschland. Nach den wissenschaftlichen Leistungen geht Rentetzi deshalb auch auf den weiteren Lebensweg der entlassenen und emigrierten jüdischen Mitarbeiterinnen des Instituts ein - am Beispiel von Marietta Blau bis in die fünfziger Jahre.

Die zahlreichen Facetten der Radioaktivitätsforschung, die Rentetzi behandelt, sind bewundernswert. Deutlich wird dabei, dass nicht nur der radioaktive Stoff, sondern auch andere materielle Ressourcen immer wieder großen Einfluss auf die Ausgestaltung wissenschaftlicher Beziehungen hatten. Es handelt sich also um ein Plädoyer für eine die materialen, ökonomischen und sozialen Bedingungen umfassende Geschichtsschreibung. Und den Wienreisenden mag das klar und leichtfüßig geschriebene Buch als Architektur- und Stadtbauführer begleiten.

Alexander von Schwerin, Braunschweig

Kohler, Robert E., 2006. All Creatures. Naturalists, Collectors, and Biodiversity, 1850-1950. Princeton, NJ: Princeton University Press, 380 S., illus. 35 \$, ISBN-13: 9780691125398.

Das jüngste Buch von Robert Kohler ist in mehrfacher Hinsicht Horizont erweiternd. Zum einen bietet es eine kulturhistorische Ergänzung des Diskurses um die Biodiversität, zum anderen handelt es von der unterbelichteten Geschichte und Theorie der Feldwissenschaften, der Erforschung von Singularität und Individualität in der „freien Natur“ auf der Spur von Pflanzen und Tieren. Während Kohler sich in seinem Buch Landscapes and Labscapes. Exploring the Lab-field Border (2002) der frühen Ökologie in Nordamerika widmete, stellt er in All Creatures Fragen nach der kulturellen Bedeutung der expansiven naturhistorischen Sammel- und Inventarisierungsaktivitäten und deren Repräsentationsformen zwischen 1850 und 1950 in den Mittelpunkt.

Im Kern bietet Kohler zwei Antworten an: Erstens sei die Entdeckung und Erforschung der Biodiversität ein modernes Phänomen, indem der Anspruch nicht nur einer systematischen, sondern einer vollständigen Erfassung biotischer Vielfalt erhoben wird; zweitens führt er vor, dass naturhistorisch motivierte Expeditionen zwischen 1880 und 1930 sowohl in Umfang wie Häufigkeit einen dramatischen Höhepunkt erreichten. Dies gilt 
zumindest für die Sammelpraktiken auf dem nordamerikanischen Kontinent beziehungsweise in den USA. Diese geographische Einschränkung wird allerdings bedauerlicherweise weder im Titel noch in der These des Buches deutlich gemacht.

Vier sozio-ökologische Komplexe macht Kohler aus: Erstens die radikale Beschleunigung der kontinentalen Landnahme durch die Einführung von dampfbetriebenen Fahrzeugen; zweitens die kulturellen Veränderungen einer Gesellschaft, die das Sammeln als Handeln an der Natur begünstigt; drittens die Entstehung staatlicher und universitärer Sammlungen, die einen Markt für naturhistorische Objekte schufen und stabilisierten, und schließlich viertens die wissenschaftliche Nobilitierung der Sammlungspraktiken durch die Taxonomie, aus der, nicht zuletzt dank der angehäuften Materialmenge, eine wissenschaftlich, institutionell und karrieretechnisch erfolgreiche Disziplin wird.

Gewissermaßen quer dazu stehen Überlegungen zu wissenschaftshistorisch eher etablierten Themen, wie zur Rollenverteilung zwischen Laien und Wissenschaftlern in der Forschung, der institutionellen Kontextualisierung von wissenschaftlichem Arbeiten in Museen und an universitären Einrichtungen, oder die Frage nach dem Status von Beobachtung in Labor und Feld. Angeschnitten, aber leider nur wenig ausgeführt wird in diesem Zusammenhang auch die Bedeutung und das Prestige der Exaktheit in den Wissenschaften. Die Naturgeschichte mit ihren Sammlungspraktiken kann zwar keine exakte Wissenschaft im Sinne einer möglichst vollständigen Mathematisierung sein, aber ihre Vertreter gehen dennoch methodisch, systematisch, empirisch und diszipliniert vor (S. 13). Schließlich sei noch ein letzter konzeptueller Baustein erwähnt, der in der Architektur des Buches eine tragende Rolle spielt, die „inner frontiers“. Sie fungieren als Scharnier zwischen disparat erscheinenden Phänomenen. An ihnen treffen Bilder von und Praktiken an der Natur in Freizeit- und Wissenschaftskultur, aber auch in Jagdund Sammleraktivitäten zusammen (S. 18). Die „innere Grenze“ bezeichnet jenen Bereich, der dann entsteht, wenn Wildnis und „zivilisierte“ Natur aufeinander stoßen und sich gegenseitig durchdringen. All dies wird im ersten Kapitel „Nature“ vorgestellt, das folglich eine Exposition des konzeptuellen Rahmens bietet, während im zweiten, „Culture“, ein historisches Panorama kultureller Praktiken der als "physische und moralische Landschaft" wiederentdeckten Natur Nordamerikas entfaltet wird, deren Identität in der vermischten Zone der „inneren Grenze“ gesucht wird (S. 48). Diese wird zu einer Landschaft für „nature-goers and nature-going“, für „fagged-out professionals and office workers" und damit zu einer für die breite Mittelklasse konsumierbaren Natur mit metaphysischer Note erklärt (S. 90).

In den folgenden drei Kapiteln - „Patrons“, „Expedition“, „Work“ - geht es um die gesellschaftlichen Bedingungen der naturhistorischen Sammelpraktiken: Welches Wissen und Material konnte aus welchen Gründen zur 
Ware werden, wer wurde von wem bezahlt und partizipierte wie an dieser Warenbörse, die geradezu eine Inflation an Objekten und Sammlern produzierte. Im vierten Kapitel untersucht Kohler, wie sich eine Expeditionskultur entfaltet, die durch inhomogene Praktiken und Akteure mit unterschiedlichem Interesse und sozialem Hintergrund charakterisiert wird (S. 180). Im fünften Kapitel diskutiert er die verschiedenen Arbeitsformen und ihre sich ändernde gesellschaftliche Bewertung - auch im Blick auf die Geschlechterordnung. Dass es nicht die Frauen, sondern die Männer waren, die schließlich eine erfolgreiche wissenschaftliche Karriere absolvierten, lag vor allem am neuen Berufsethos der Mittelklasse: Die Grenze zwischen Profession und Freizeit hatte sich auch in der Praxis des Sammelns verhärtet, die Frauen waren dabei auf die Seite der Akteure geraten (S. 220). Die „richtige“ Seite wird im sechsten Kapitel "Knowledge“ diskutiert, das der taxonomischen Arbeit im akademischen Umfeld gewidmet ist (S. 270). Das Problem der Unterscheidung und Benennung von Arten, überhaupt von Einheiten und Kategorien analysiert Kohler an Beispielen aus der Genetik und der Taxonomie. Auch hier steht das Verhältnis zwischen wissenschaftlicher Praxis und Repräsentation im Mittelpunkt seiner Untersuchung, ohne dass der Autor einen Hinweis auf tiefer anzulegende wissenschaftsphilosophische Fragen gibt, wie etwa nach der Herstellung von Evidenz durch Visualisierung oder dem hier involvierten Nominalismus-Realismus Problem.

Kohlers Buch bietet einen produktiven Anschluss an so unterschiedliche Studien wie etwa Idea of Biodiversity. Philosophies of Paradise (Takacs 1996), Nature's Economy (Worster 1977) oder Cultures of Natural History (Jardine/ Secord/Spary 1996). Das auch an Bildern reiche Buch lädt mit seinem überzeugenden methodischen Zugriff zur Reflexion auf die Reichweite und Leistungsfähigkeit kulturhistorischer Ansätze ein und damit gleichermaßen zum Verhältnis von Wissenschaftsgeschichte und Wissenschaftsforschung. Nicht zuletzt trägt die leise im Hintergrund kommentierende, zuweilen mahnend anschwellende Stimme des Autors zur Intensität des Leseerlebnisses bei. Sie erinnert uns immer wieder daran, dass wir unsere Mitgeschöpfe besser verstehen lernen müssen, da unsere eigene Zukunft von ihnen abhängt.

Astrid E. Schwarz, Darmstadt

Sommer, Marianne, 2007. Bones \& Ochre. The Curious Afterlife of the Red Lady of Paviland. Cambridge, Mass.: Harvard University Press, geb. 416 S., 32,99 €, ISBN-13: 978-0-674-02499-1.

1823 fand William Buckland in einer Höhle an der südwalisischen Küste ein kopfloses Skelett, dessen Knochen von Ocker rötlich gefärbt waren. Bones $\mathcal{E}$ Ochre hat Marianne Sommer ihre Zürcher Habilitationsschrift genannt. Darin rekonstruiert sie The Curious Afterlife of the Red Lady of Paviland, 
so der Untertitel, anhand der drei Interpretationen von William Buckland (1784-1856), William Sollas (1849-1936) und Stephen Aldhouse-Green.

Im ersten Teil zeigt die Autorin, wie der Oxford-Geologe und anglikanische Geistliche Buckland sich selbst als romantischen, intuitiven und genialen Wissenschaftler inszenierte und damit auch mit Humor gegen Kritik immunisierte. Der große Geschichtenerzähler machte aus seinem Fund eine Hexe aus römischer Zeit. Er versuchte mit all seiner Autorität zu zeigen, dass die Red Lady kein vorsintflutlicher Mensch war, denn das hätte nicht zu seiner natürlichen Theologie gepasst. Im Pariser Akademiestreit schlug sich Buckland auf Cuviers Seite, denn die Wandelbarkeit der Arten stand im Widerspruch zu seinem Weltbild.

Sommer geht es dabei in jedem ihrer drei Kapitel um mehr als nur die Beschäftigung ihres jeweiligen Protagonisten mit der Red Lady. Im zweiten Teil ihres Buches spielt die Dame, die mittlerweile als Mann gilt, gar eine untergeordnete Rolle, denn hier legt sie den Fokus auf die großen anthropologischen Debatten des ersten Drittels des 20. Jahrhunderts um die Hierarchie der "Rassen“ und Modelle der Migration und der Verdrängung. Der zweite Oxforder Geologe ihrer Geschichte, William Sollas, sah heutige „Rassen“ gar als Nachfahren paläolithischer Spezies an. Imperialismus und Erster Weltkrieg erwiesen sich als prägend für die Forschung zu den menschlichen Ursprüngen der Zeit. Der vermeintlich ungeschlachte Neandertaler und der „Wohlgestaltete" Cro-Magnon-Mensch (der heute als früher Homo sapiens gilt) wurden dabei immer stärker kontrastiert. So erklärte der französische Anthropologe Marcellin Boule sie sogar einmal zu Vorfahren von Deutschen beziehungsweise Franzosen. Sollas selbst interpretierte die Red Lady als CroMagnon.

Im dritten Teil dekonstruiert die Autorin den Anspruch eines „definitiven Berichts" über die Red Lady durch den walisischen Archäologen Stephen Aldhouse-Green in seinem im Jahre 2000 herausgegebenen Sammelband. Gerade die Rede von der Internationalität und der Interdisziplinarität der Forschung, die ja nicht nur Paläoanthropologen wie eine Monstranz vor sich hertragen, erweist sich mehr als Rhetorik denn als Realität. So wurde die Red Lady als „Urwaliserin“ vereinnahmt und die Forderung erhoben, die Fossilien, die noch immer in Oxford lagern, an ihr „Herkunftsland“ zurückzugeben. Derartige Aussagen kommen im 21. Jahrhundert zwar eher von vorwortschreibenden Politikern denn von Forschern, sie zeigen aber, dass Fossilien nach wie vor noch dazu dienen, nationale Identitäten zu konstruieren.

Sommer spricht nicht von Paläoanthropologie, sondern genauer von der Human-Origins-Forschung, also jenem Konglomerat aus Paläoanthropologie, Archäologie, Geologie, Paläogenetik und anderen Disziplinen, deren Vertreter „unsere“ Herkunft entschlüsseln und ein Paradebeispiel für Interdisziplinarität sein wollen. Wie aber nicht nur das Beispiel der Red Lady 
zeigt, bestehen nach wie vor Spannungen zwischen morphologisch arbeitenden Paläoanthropologen und Vertretern molekularbiologischer Methoden, ob die Wahrheit in den Fossilien oder in der DNA zu finden ist.

Die geleistete Kontextualisierungsarbeit ist enorm und verlangt den Leserinnen und Lesern gelegentlich etwas Durchhaltevermögen ab. Was macht den Erkenntnisgewinn dieser Knochenarbeit aus? Sommer arbeitet Denkmuster heraus, die meist in binären Gegensätzen bestehen, etwa Kontinuität oder Verdrängung, Mono- oder Polygenese, „Human Antiquity“ oder rezenter Ursprung des Menschen. Die Autorin redet damit aber nicht einer ahistorisch-strukturalistischen Sichtweise das Wort, nach der die Interpretationsmuster begrenzt und letztlich immer dieselben sind. In Sommers epistemologischem Modell kommen stets neue hinzu, wie etwa die Spannung zwischen morphologischen und molekularbiologischen Ansätzen. Vor allem aber können die überlieferten Konzepte je nach Kontext sehr unterschiedlich angewandt und kombiniert werden, sind also alles andere als statisch. Bones $\mathcal{E}$ Ochre ist eine gelungene Fallstudie, gerade weil sie auch mit grundsätzlichen Einsichten zur Human-Origins-Forschung aufwartet.

P.S.: Die Geschichte ist nicht abgeschlossen: 2008 ergab eine Neudatierung der Red Lady, dass sie vor gut 29.000 Jahren lebte und nicht vor etwa 26.000 Jahren, wie vor acht Jahren im „definitiven Report“ behauptet.

Oliver Hochadel, Barcelona

Sarasin, Philipp/Berger, Silvia/Hänseler, Marianne/Spörri, Myriam, Hg., 2007. Bakteriologie und Moderne. Studien zur Biopolitik des Unsichtbaren 1870-1920. Frankfurt a.M.: Suhrkamp, 529 S., $17 €$, ISBN-13: 978-3518294079.

Das Buch ist ein Zwischending aus Aufsatzband und Quellenedition. Insgesamt dreizehn Beiträge zur Wissenschaftsgeschichte der Bakteriologie laden zur Lektüre ein, zehn von ihnen wurden aus dem Amerikanischen oder Englischen, einer wurde aus dem Französischen übersetzt. Die Herausgeberinnen und der Herausgeber sprechen von einer „Sammlung von Lesefrüchten" und haben diese mit einer programmatischen Einleitung versehen.

Gemeinsam mit den vorangestellten Überlegungen wird das Gesamtpaket präsentiert als eine Art Zwischenbilanz des wissenschaftsgeschichtlichen Forschungsstands zur Bakteriologie. Dokumentiert werden soll aber auch die Leistungsfähigkeit „jener neueren Wissenschaftsgeschichte, die seit den 1960er Jahren begonnen hat, das traditionelle Bild der Entwicklung einer Wissenschaft als einer sich sukzessive entfaltenden, sicher fortschreitenden, kumulativen und teleologisch ausgerichteten Rationalität fundamental zu erschüttern“ (S. 9). Wissensgeschichte als "Genealogie“ - mit dieser Pro- 
grammformel wird ausdrücklich an Foucault angeknüpft: Die Herausgeber halten die Relativierung von Wahrheitsansprüchen sowie den Verzicht auf Entwicklungs- und Ursprungsdenken als methodologische Eckpunkte für die Wissenschaftsgeschichte fest. Im Hinblick auf den exemplarischen Fall der Bakteriologie betonen sie außerdem die interdiskursiven Bezüge, die "politisch-imaginäre Dimension" der bakteriologischen Theorien und Praktiken, deren „biopolitische Wirksamkeit“. Und sie bieten eine Leitfrage an, unter der sich die Beiträge des Bandes gewinnbringend lesen lassen: Inwiefern bestehen Beziehungen zwischen dem "bakteriologischen Paradigma“ (S. 15) und einer nicht nur als Zeitabschnitt, sondern auch als systematisches Unterfangen verstandenen Moderne? Was macht aus der wissenschaftlichen Bakteriologie vielleicht sogar ein spezifisch modernes Projekt?

Die Beiträge von Owsei Temkin (zum Infektionsbegriff), Erwin $\mathrm{H}$. Ackerknecht, Bruno Latour, J. Andrew Mendelsohn, Gerald L. Geison (mit je verschieden gelagerten Analysen zum Spannungsfeld zwischen antikontagionistischer Hygiene, den Bakteriologien Pasteurs und Kochs sowie der nachfolgenden Epidemiologie), Mariko Ogawa (zur britischen Reaktion auf Kochs Theorie des Cholerabazillus), Christoph Gradmann sowie Paul Weindling (zur deutschen Bakteriologie im Kaiserreich und bis 1920), Alexandra Minna Stern (zur bakteriologischen Dimension des amerikanischmexikanischen Grenzregimes bis 1930), Philipp Sarasin (zu technischen und sprachlichen Übertragungseffekten - „Metaphern“ - in der Bakteriologie), Warwick Anderson (über das Konzept der rassenspezifischen Immunität in der britischen Tropenmedizin bis 1920) und abschließend Marouf A. Hasian (zum Fallbeispiel der Typhusüberträgerin „Typhoid Mary“) sind durchweg instruktiv und - nicht zuletzt im Blick auf die Leitfrage - spannend zu lesen.

Nachdenklich macht allerdings die Chronologie des Bandes, denn zum einen beziehen sich die Beiträge (trotz des Untertitels) auf einen Zeitraum von etwa 1820 bis mindestens 1930, zum anderen datieren sie auf 1948 bis 2007, sind also ihrerseits zu deutlich unterschiedlichen Zeiten entstanden und verkörpern über diesen langen Zeitraum hinweg deutlich spürbar einen Wandel der Zugangsweisen. Letztlich fragt man sich, was für eine Geschichte der Band denn nun besser beleuchtet: diejenige der Bakteriologie oder diejenige ihrer Geschichtsschreibung. Die Herausgeberinnen und der Herausgeber haben, wenn man so will, dann doch der Historie den Vorzug gegeben und die Chronologie der Gegenstandsebene gewählt. So folgt auf einen Beitrag von 1953/1977 einer von 1948, dann folgen weitere von 1984, 2003, 2001, 1996, 2000. Der aktuellste Beitrag (von Sarasin selbst) stammt aus dem Jahr 2004. Dies legt nun das reizvolle Gedankenspiel nahe, die Verhältnisse umzukehren, also alle Beiträge nach dem Entstehungsdatum zu ordnen und den Band als eine bloße Variation des Themas Bakteriologie zu lesen, während in der Hauptsache dann - einschließlich des Vorwortes - die 
Wissenschaftsgeschichtsschreibung bei der allmählichen Verfertigung ihrer Gedanken und Formen zu beobachten ist.

Gewisse editorische Schwächen stören, so wurden Nachweise auf im Original deutschsprachige Quellen nicht durchgehend umgestellt, und hier und da hakt die Übersetzung. Gleichwohl ist der Band nützlich, enthält reichhaltige Anregungen und ist als Einführung ins Thema - etwa für Studierende - geradezu ideal.

Petra Gehring, Darmstadt 